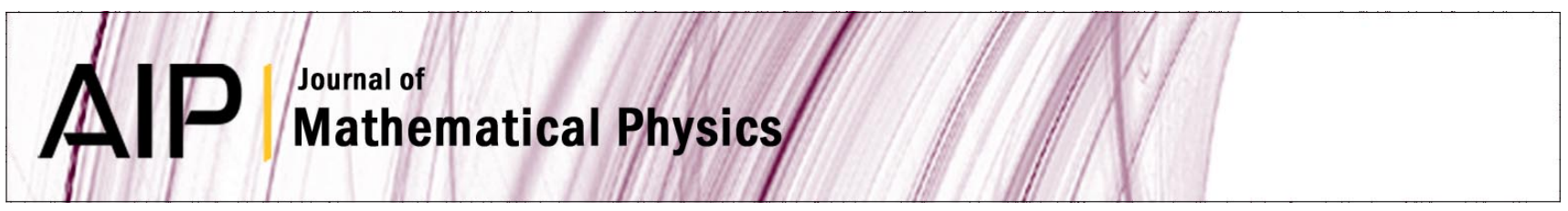

\title{
Operator extension of strong subadditivity of entropy
}

Isaac H. Kim

Citation: J. Math. Phys. 53, 122204 (2012); doi: 10.1063/1.4769176

View online: http://dx.doi.org/10.1063/1.4769176

View Table of Contents: http://jmp.aip.org/resource/1/JMAPAQ/v53/i12

Published by the American Institute of Physics.

\section{Related Articles}

A stochastic optimization approach to coarse-graining using a relative-entropy framework J. Chem. Phys. 138, 044313 (2013)

Entropy of polydisperse chains: Solution on the Husimi lattice J. Chem. Phys. 138, 044902 (2013)

Impacts of length and geometry deformation on thermal conductivity of graphene nanoribbons J. Appl. Phys. 113, 044306 (2013)

Molecular dynamic simulation of diamond/silicon interfacial thermal conductance J. Appl. Phys. 113, 024907 (2013)

Influence of chaotic synchronization on mixing in the phase space of interacting systems Chaos 23, 013103 (2013)

\section{Additional information on J. Math. Phys.}

Journal Homepage: http://jmp.aip.org/

Journal Information: http://jmp.aip.org/about/about_the_journal

Top downloads: http://jmp.aip.org/features/most_downloaded

Information for Authors: http://jmp.aip.org/authors

\section{ADVERTISEMENT}

The most comprehensive support for physics in any mathematical software package World-leading tools for performing calculations in theoretical physics

www.maplesoft.com/physics
Your work in Maple matches how you would write the problems and solutions by hand

- State-of-the-art environment for algebraic computations in physics

The only system with the ability to handle a wide range of physics computations as well as pencil-and-paper style input and textbook-quality display of results

- Access to Maple's full mathematical power, programming language, visualization routines, and document creation tools 


\title{
Operator extension of strong subadditivity of entropy
}

\author{
Isaac H. Kim \\ Institute of Quantum Information, California Institute of Technology, Pasadena, \\ California 91125, USA
}

(Received 22 October 2012; accepted 5 November 2012; published online 30 November 2012)

We prove an operator inequality that extends strong subadditivity of entropy: after taking a trace, the operator inequality becomes the strong subadditivity of entropy. (C) 2012 American Institute of Physics. [http://dx.doi.org/10.1063/1.4769176]

\section{INTRODUCTION}

There are inequalities satisfied by Von Neumann entropy, such as non-negativity and strong subadditivity. ${ }^{1,2}$ Strong subadditivity plays an important role in quantum information theory: it is essential in virtually all nontrivial coding theorems. Strong subadditivity has also found applications to geometric entropy, ${ }^{3}$ conformal field theory (CFT), ${ }^{4}$ and topological entanglement entropy. ${ }^{5}$

In this paper, we prove a certain operator inequality that extends strong subadditivity. Motivation for studying such inequality comes from recent observation of $\mathrm{Li}$ and Haldane (LH). ${ }^{6} \mathrm{LH}$ conjectured and numerically substantiated that eigenvalues of reduced density matrix contains universal information about the quantum phase. While their result concerns a variational wavefunction for a fractional quantum Hall system, author was able to reproduce some of the consequences without resorting to the underlying structure of the wavefunction dictated by the CFT. ${ }^{7}$

One important assumption was that certain operator generalization of strong subadditivity holds for general quantum states. Specifically, the author conjectured the following inequality,

$$
\operatorname{Tr}_{A B}\left(\rho_{A B C}\left(\hat{H}_{A B}+\hat{H}_{B C}-\hat{H}_{B}-\hat{H}_{A B C}\right)\right) \geq 0,
$$

where $\hat{H}_{A}$ is formally defined as

$$
\hat{H}_{A}=-I_{A^{c}} \otimes \log \left(\rho_{A}\right) .
$$

Other operators are defined similarly. Whenever logarithm of reduced density matrix appears, tensor product with identity for the rest of the subsystems is implicitly assumed. We prove the conjecture in this paper.

\section{OPERATOR GENERALIZATION OF STRONG SUBADDITIVITY}

Since Lieb and Ruskai's seminal result, ${ }^{1}$ alternative proofs for strong subadditivity have been introduced by several authors. ${ }^{8-11}$ In particular, Effros recently presented a proof based on perspective of operator convex function. ${ }^{11}$ Effros basically extended the notion of perspective function from real numbers to operators. Given a function $f$, perspective of $f$ is defined as

$$
g(x, t)=f(x / t) t .
$$

If $f(x)$ is convex, $g(x, t)$ is jointly convex in $x$ and $t$. Main insight of Effros is that similar statement holds for function $f$ that is operator convex. To be more precise, he showed the following statement.

Theorem 1: $\left(\right.$ Effros $\left.^{11}\right)$ If $f(x)$ is operator convex, and $[L, R]=0$, perspective

$$
g(L, R)=f(L / R) R
$$


is jointly convex in the sense that if $L=c L_{1}+(1-c) L_{2}$ and $R=c R_{1}+(1-c) R_{2}$ with $\left[L_{i}, R_{i}\right]$ $=0(i=1,2), 0 \leq c \leq 1$,

$$
g(L, R) \leq \operatorname{cg}\left(L_{1}, R_{1}\right)+(1-c) g\left(L_{2}, R_{2}\right) .
$$

We choose a matrix algebra $\mathcal{B}(\mathcal{H})$ with an inner product structure of $\langle X, Y\rangle=\operatorname{Tr}\left(X Y^{\dagger}\right)$, where $X, Y$ are $n \times n$ matrices. Following Effros, we choose $L$ and $R$ to be superoperators that multiplies matrix from left or right. For $X \in \mathcal{B}(\mathcal{H}), L$ and $R$ are defined as follows.

$$
\begin{aligned}
& L X=\rho X, \\
& R X=X \sigma .
\end{aligned}
$$

$L$ and $R$ commute with each other. One can also show the following relations.

$$
\begin{aligned}
& \log (L) X=\log (\rho) X, \\
& \log (R) X=X \log (\sigma) .
\end{aligned}
$$

Effros' result implies the following statement.

Theorem 2:

$$
\operatorname{Tr}_{A B}\left(\rho_{A B C}\left(\hat{H}_{A B}+\hat{H}_{B C}-\hat{H}_{B}-\hat{H}_{A B C}\right)\right) \geq 0
$$

Proof: Let $f(x)=x \log x$. Since $f(x)$ is operator convex, ${ }^{12} g(L, R)=L \log (L)-L \log (R)$ is jointly convex in $L$ and $R$. Therefore,

$$
\langle g(L, R)(O), O\rangle=\operatorname{Tr}\left(\rho \log \rho O O^{\dagger}-\rho O \log \sigma O^{\dagger}\right)
$$

is jointly convex in $L$ and $R$ for all $O \in \mathcal{B}(\mathcal{H})$. Joint convexity of Eq. (9) was originally proved by Petz. ${ }^{14}$ Choose $\rho=\rho_{A B C}, \sigma=\rho_{A B} \otimes \frac{I_{C}}{d_{C}}, O=I_{A B} \otimes P_{C}$, where $P_{C}$ is a projector supported on $C$ and $d_{C}$ is the dimension of $C$. Note

$$
\frac{I_{A}}{d_{A}} \otimes \rho_{B C}=\frac{1}{d_{A}^{2}} \sum_{i=1}^{d_{A}^{2}} U_{A, i} \rho_{A B C} U_{A, i}^{\dagger}
$$

for a set of unitaries $\left\{U_{A, i}\right\}$ that forms an orthogonal basis for $\mathcal{B}\left(\mathcal{H}_{A}\right)$. An example can be found in Ref. 13. Using joint convexity,

$$
\operatorname{Tr}\left(\frac{I_{A}}{d_{A}} \otimes \rho_{B C}\left(\hat{H}_{B}-\hat{H}_{B C}\right) P_{C}\right) \leq \operatorname{Tr}\left(\rho_{A B C}\left(\hat{H}_{A B}-\hat{H}_{A B C}\right) P_{C}\right) .
$$

Left-hand side of the inequality is equal to $\operatorname{Tr}\left(\rho_{A B C}\left(\hat{H}_{B}-\hat{H}_{B C}\right) P_{C}\right)$. Since the inequality holds for all $P_{C}$,

$$
\operatorname{Tr}_{A B}\left(\rho_{A B C}\left(\hat{H}_{A B}+\hat{H}_{B C}-\hat{H}_{B}-\hat{H}_{A B C}\right)\right) \geq 0
$$

One may wish to find a similar inequality when partial trace is restricted to $A$ or $B$. In both cases, the resulting operators are not even Hermitian.

\section{CONCLUSION}

We have proved an operator extension of strong subadditivity conjectured in Ref. 7. While our method was based on the operator convexity of the function $f(x)=x \log x$, Effros' approach can be generalized to other operator convex functions as well. It would be interesting to find applications of these new family of operator inequalities. 


\section{ACKNOWLEDGMENTS}

This research was supported in part by NSF (Grant No. PHY-0803371), by ARO (Grant No. W911NF-09-1-0442), and DOE (Grant No. DE-FG03-92-ER40701). I would like to thank Alexei Kitaev for introducing Effros' work.

${ }^{1}$ E. H. Lieb and M. B. Ruskai, "Proof of the strong subadditivity of quantum-mechanical entropy," J. Math. Phys. 14(12), 1938-1941 (1973)

${ }^{2}$ M. A. Nielsen and I. L. Chuang, Quantum Computation and Quantum Information, Cambridge Series on Information and the Natural Sciences (Cambridge University Press, 2000).

${ }^{3}$ H. Casini, "Geometric entropy, area, and strong subadditivity," Class. Quantum Grav. 21, 2351-2378 (2004).

${ }^{4}$ H. Casini and M. Huerta, "A finite entanglement entropy and the c-theorem," Phys. Lett. B 600, 142-150 (2004).

${ }^{5}$ T. Grover, A. M. Turner, and A. Vishwanath, "Entanglement entropy of gapped phases and topological order in three dimensions," Phys. Rev. B 84, 195120 (2011).

${ }^{6} \mathrm{H}$. Li and F. D. M. Haldane, "Entanglement spectrum as a generalization of entanglement entropy: Identification of topological order in non-abelian fractional quantum Hall effect states," Phys. Rev. Lett. 101, 010504 (2008).

${ }^{7}$ I. Kim, "Determining structure of real-space entanglement spectrum from approximate conditional independence," preprint arXiv:1210.1831 (2012).

${ }^{8}$ D. Petz, "Monotonicity of quantum relative entropy revisited," Rev. Math. Phys. 15, 79 (2003).

${ }^{9}$ M. A. Nielsen and D. Petz, "A simple proof of the strong subadditivity inequality," preprint arXiv:quant-ph/0408130 (2004).

${ }^{10}$ M. B. Ruskai, “Another short and elementary proof of strong subadditivity of quantum entropy,” Rep. Math. Phys. 60 , $1-12(2007)$

${ }^{11}$ E. G. Effros, "A matrix convexity approach to some celebrated quantum inequalities," Proc. Natl. Acad. Sci. U.S.A. 106(4), 1006-1008 (2009).

${ }^{12}$ R. Bhatia, Matrix Analysis, Graduate Texts in Mathematics (Springer, 1997).

${ }^{13}$ A. O. Pittenger and M. H. Rubin, "Separability and Fourier representations of density matrices," Phys. Rev. A 62, 032313 (2000).

${ }^{14}$ D. Petz, “Quasi-entropies for finite quantum systems," Rep. Math. Phys. 23, 57-65 (1986). 